\title{
Review of safety improvement on sodium-cooled fast reactors after Fukushima accident
}

\author{
Toshikazu Takeda $^{1^{*}}$, Yoichiro Shimazu $^{1}$, Basma Foad $^{1,2}$, Katsuhisa Yamaguchi $^{1}$ \\ ${ }^{1}$ Research Institute of Nuclear Engineering, University of Fukui, Fukui, Japan; ${ }^{*}$ Corresponding Author: $\mathrm{t}$ takeda@u-fukui.ac.jp \\ ${ }^{2}$ Egyptian Nuclear and Radiological Regulatory Authority, Cairo, Egypt
}

Received 28 September 2012; revised 30 October 2012; accepted 12 November 2012

\begin{abstract}
Several countries are developing and deploying SFRs even after the accident at Tokyo Electric Power Company's Fukushima Dai-Ichi Nuclear Power Station. However, the Fukushima accident prompted all countries to redefine the fast reactor programs. The drastic safety enhancement is the most important issue to be established. In light of this situation, key essence of the safety improvement is reviewed in this paper by referring the achievements of the recent International Workshop on Prevention and Mitigation of Severe Accidents in SFRs which was held by Japan Atomic Energy Agency (JAEA) in cooperation with the International Atomic Energy Agency (IAEA) in June, 2012 and the findings published in the past journals including those of the International Conference on Fast Reactor and Related Fuel Cycles (FR09) held by IAEA in December, 2009.
\end{abstract}

Keywords: Safety; Improvement; Fast Reactors; Fukushima Accident

\section{INTRODUCTION}

Nuclear reactors have been developed since 1950's in various countries because they can deliver huge amounts of energy to both emerging and developed economies. The importance of nuclear energy, as a realistic option to solve the issues of the depletion of energy resources and the global environment, has been acknowledged worldwide. However, acceptance of large scale contributions would depend on satisfaction of key drivers to enhance sustainability in terms of economics, safety, adequacy of natural resources, waste reduction, non-proliferation and public acceptance. Fast reactors with fuel recycle enhance the sustainability indices significantly. This has led to the focus on Sodium-Cooled Fast Reactors (SFR) in the Generation IV International Forum (GIF) and the
International Project on Innovative Nuclear Reactors and Fuel Cycles (INPRO) initiative of the International Atomic Energy Agency (IAEA).

New fast reactors are expected to be commissioned in the near future in the Russian Federation. Moreover, China, France, India, Japan, Republic of Korea and the United States of America are preparing advanced prototypes/demonstrations and/or commercial reactors for the 2020-2030 horizon. The necessary condition for successful fast reactor deployment is the understanding and assessment of innovative technological and design options, based on both past knowledge and experience, as well as on ongoing research and technology development efforts. In this respect, IAEA convened, on 7-11 December 2009 in Kyoto, Japan, the International Conference on "Fast Reactors and Related Fuel CyclesChallenges and Opportunities (FR09)", hosted by the Japan Atomic Energy Agency (JAEA). This conference aims at promoting the exchange of information on national and multinational programmes and new developments and experience, with the goal of identifying and critically reviewing problems of importance, and stimulating and facilitating cooperation, development and successful deployment of fast reactors in an expeditious manner.

The severe accident at Tokyo Electric Power Company's Fukushima Dai-Ichi Nuclear Power Station caused by the Great East-Japan Earthquake and Tsunami on March 11, 2011 prompted all countries to redefine the fast reactor programs. In order to achieve the successful deployment of fast reactors, the drastic safety enhancement is the most important issue to be established, especially for Japan where the restart of nuclear power plants once being stopped is a serious matter of argument. In light of this situation, JAEA held an International Workshop on "Prevention and Mitigation of Severe Accidents in SFRs" in cooperation with IAEA at the Wakasa Wan Energy Research Center in Tsuruga on 12-13 June, 2012. In this workshop, safety improvement approaches for SFRs after the Fukushima accident were illustrated from several countries. 
Key essence of the safety improvement is reviewed in this paper by referring the achievements of this workshop and the findings published in the past journals including the Special Issue of the "Journal of Nuclear Science and Technology" which compiles globally valued papers presented at the FR09 conference, and summarized in Section 2. Also the IAEA's activities are summarized in Section 3. Finally the key characteristics for the safety improvement of SFR is concluded in Section 4.

\section{EXAMPLES OF SAFETY APPROACH IN COUNTRIES DEVELOPING FAST REACTORS}

\subsection{Japanese Approach}

In Japan, there is an experimental SFR Joyo, and a prototype fast reactor Monju. As for these existing SFR systems, safety designs and assessments have been carried out from the early stage of their development, by taking into account, in particular, prevention and mitigation of the severe accidents on the same basis as the IAEA's current Defense in Depth (DiD) principles shown in Table 1 [1].

After the Fukushima accident, additional safety measures, similar to light water reactors in Japan, have been employed in Monju [2]. The Monju plant is placed at 21 $\mathrm{m}$ higher than the sea level, so there will be no problem for the tsunami attack. Furthermore, in the case of Station Black-Out (SBO), the decay heat is transferred to the air cooler of the secondary cooling system by using the natural convention of sodium in the primary and secondary cooling systems, and the transferred heat is removed from the air cooler by diffusing the heat to atmosphere by air. The use of Monju for the safety research and prevention and mitigation of severe accidents in SFRs is essential [3]. In particular, experiments on natural circulation are extremely precious for the safety assurance of SFRs.

Japanese Sodium-cooled Fast Reactor (JSFR) has been proposed as the next generation (Generation-IV) fast reactor. Before the Fukushima accident, it was planned that a demonstration JSFR will start from 2025 and a commercial type JSFR of $1500 \mathrm{M}$. We will start from 2050. JAEA also proposed the SFR Safety Design Criteria (SDC) for achieving safety goals by applying harmonized safety approaches [4]. General features of the safety design requirements are: 1) Achievement of higher reliability; 2) Achievement of higher inspect-ability and maintainability; 3) Introduction of passive safety features; 4) Reduction of operator action needs; 5) Design consideration against Beyond Design Basis Accidents (BDBA); 6) In-vessel retention of degraded core materials; 7) Prevention and mitigation against sodium chemical reaction; and 8) Design against external events [5].

Several innovative techniques have been developed for the improvement in economics, safety and reliability of JSFR [6]. For the economics, important techniques are developed such as two cooling loops for the system simplicity, compact reactor containment of high-chromium steal to shorten pipe development and high-burn-up fuel. Also for the safety and reliability improvement, several ideas are included such as the use of passive reactor shut-down system, and the core cooling by natural sodium circulation in case of SBO and the use of special fuel assemblies with inner duct to present re-criticality in case of severe accidents.

Elimination of the severe power burst events in the Core Disruptive Accident (CDA) is intended [7]. The design strategy for it is to control the potential of excessive void reactivity insertion in the initiating phase of CDA by selecting appropriate design parameters such as maximum void reactivity on one hand, and to exclude core-wide molten-fuel-pool formation by introducing an inner duct in a fuel subassembly as shown in Figure 1 to discharge molten fuel from the core on the other hand. Figure 2 shows an example of the early discharge of the molten fuel of about $20 \%$ during the Unprotected Loss of Flow (ULOF) accident.

By analyzing ULOF accidents, the core height of less than $1.0 \mathrm{~m}$ was selected and the maximum sodium void reactivity was suppressed under $6 \$$. Limiting the core height contributes to provide an effective fuel dispersal reactivity change. For the reactor core with the sup-

Table 1. IAEA's defense-in-depth principles.

\begin{tabular}{cll}
\hline & \multicolumn{1}{c}{ Level of IAEA's Defense-In-Depth } & Plant State \\
\hline Level-1: & Prevention of abnormal operation and failures & Normal operation \\
Level-2: & Control of abnormal operation and detection of failures & Anticipated Operational Occurrence (AOO) \\
Level-3: & Control of accidents within the design basis & Design Basis Accident (DBA) \\
Level-4: & $\begin{array}{l}\text { Control of severe plant conditions, including prevention of accident progression } \\
\text { and mitigation of the consequences of severe accidents }\end{array}$ & $\begin{array}{l}\text { Design Extension Conditions (DEC) - } \\
\text { including significant core degradation }\end{array}$ \\
Level-5: & $\begin{array}{l}\text { Mitigation of radiological consequences of significant releases of radioactive materials } \\
\text { Corresponds to offsite emergency response }\end{array}$ &
\end{tabular}




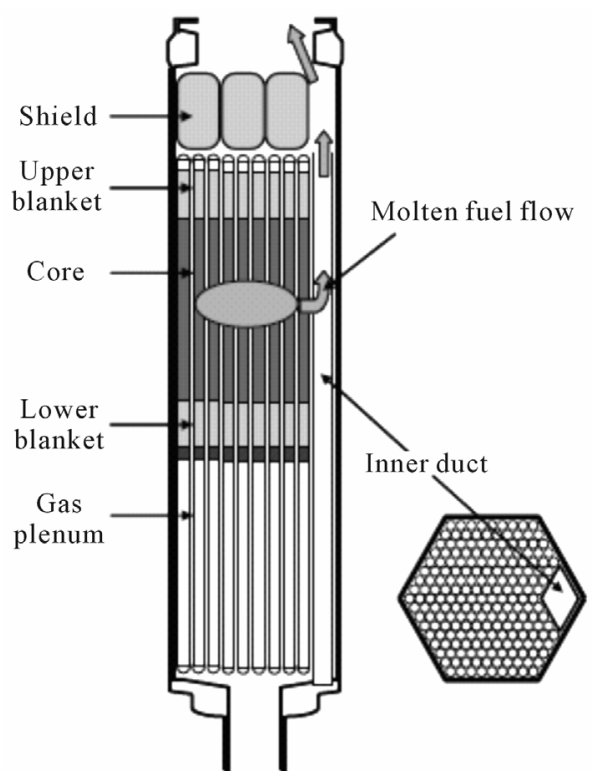

Figure 1. Fuel subassembly concept with inner duct structure [5].

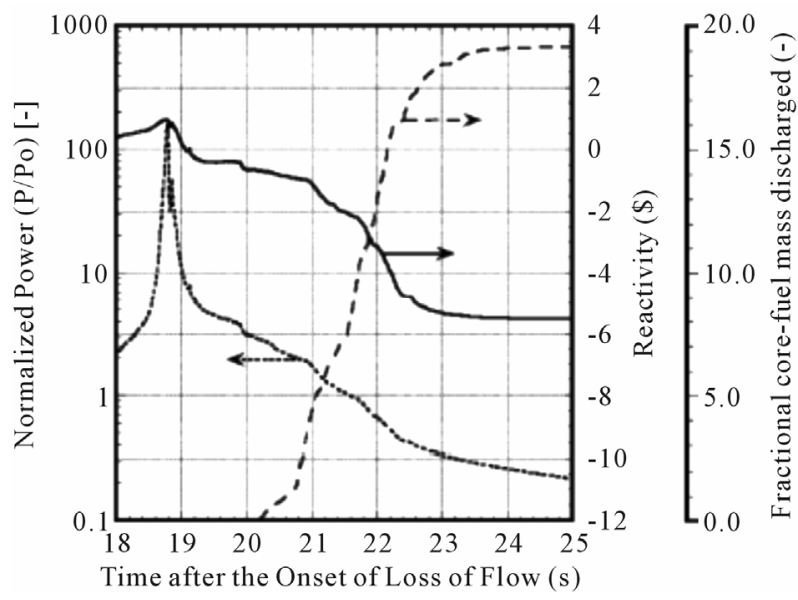

Figure 2. Early fuel discharge evaluated for JSFR [7].

pressed sodium void reactivity, the released energy can be retained within the reactor vessel.

Besides JSFR and relating activities, the $4 \mathrm{~S}$ reactor

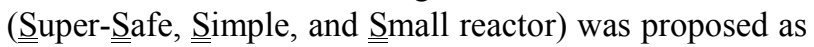
the electric and heat supply in rural districts. The 4 S reactor is a sodium-cooled reactor, and it can operate 30 40 years without fuel exchange. The burn-up reactivity loss during 30 - 40 years is compensated by driving the movable reflectors up and down. The neutron leakage rate is adjusted by the reflector motion. The sodium void reactivity is of course negative. In addition to the inherent safety features, there are two independent systems for reactor shutdown. The primary shutdown system provides for a drop of several sectors of the reflector, and the back-up shutdown system provides for insertion of the ultimate shutdown rod, located as a central subas- sembly on a stand-by in a fully out condition $[8,9]$.

\subsection{French Approach}

In France, there is a plan to build the ASTRID reactor illustrated in Figure 3. The ASTRID design studies, full scale compartment testing, feasibility study for the fabrication of minor actinides bearing experimental fuel, and core fabrication workshop are in progress in addition to severe accidents experimental program $[10,11]$. ASTRID is the pool-type SFR which has favorable intrinsic features to cool the reactors such as a large thermal inertia, diversified heat sink, natural circulation, ability to guarantee minimum sodium level. The large sodium quantity of primary coolant provides for a high thermal inertia in case of loss of main heat sink. And good natural convection and circulation features allow to design passive, diversified decay heat removal systems.

The main design issues concerning severe accident mitigation are the minimization of the mechanical energy release, consideration of molten fuel-coolant interaction consequence, robustness of containment, installation of core catcher, and so on. The core catcher is investigated to locate at several positions. The purpose is to maintain the core debris in a safe state, where sub-criticality and decay heat removal are maintained.

The previous studies illustrated the sodium void effect for limiting the mechanical energy release. The mitigation of core melt-down consequences with sodium void effect higher than about $5 \$$ is not reasonably achievable [12]. As for the core reactivity issue, Research and Development (R \& D) of core configuration with overall negative sodium void coefficient has been performed. As one of the option, the heterogeneous cores were investigated. It is noted that the assessment of the accident scenario during ULOF has some uncertainty because calculation

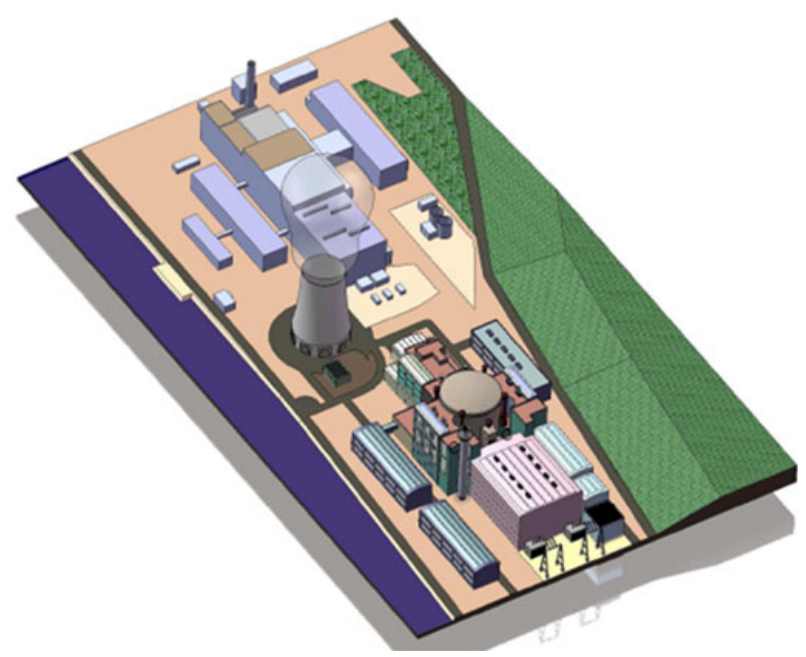

Figure 3. Bird's-eye view of ASTRID (after FR09 presentation handout). 
results are very sensitive to key parameters and modeling of accidents, and the results of a phase is very much dependent to the previous phase. Thus it is important to consider the uncertainty of the accident scenario.

\subsection{Russian Approach}

In Russia, two fast reactors are operating now: the test reactor BOR-60 and the fast reactor BN-600. The research reactor BR-10 is on preparation for decommissioning, and the SFR BN-800 is under construction. The large size SFR BN-1200 will be constructed, and now the design is developing. In the safety assessment for BN-1200, the IAEA's DiD principle [13] up to fifth level is applied. In the fourth level, prevention of core damage under severe BDBA conditions is based on both inherent safety and two types of passive safety systems. By using these systems, probability of the core damage decreases by at least one order of magnitude as compared to regulatory requirements of down to $10 \mathrm{E}-6 /$ reactor year [14].

The regulatory authority in Russia presented technical proposals for aiming the safety improvement for reactor core, reactor facility, monitoring, control, safety and diagnostic systems and so on. For example, the minimization of sodium void reactivity effect for the reactor core and the use of automatic shut-down system without safety system actuation for reactor facility are proposed. The requirement for the sodium void reactivity effect was changed after Chernobyl accident to such that the integral sodium void reactivity effect is near zero. To minimize the sodium void reactivity effect, the following analyses were performed using the following design changes [15]:

1) Arrangement of sodium plenum above the core; sodium void in the plenum gives negative reactivity;

2) Decrease of core height; a well known design to reduce the sodium void reactivity effect;

3) Annular or modular core and etc.;

4) Adding moderator to the reactor.

These design changes result in both advantage and disadvantage, where the former gives negative void reactivity coefficient and the latter gives deterioration of technical and economical characteristics.

Concept 1) was adopted in BN-800 reactor design, and combined concept 1) +2 ) is proposed for BN-1200 type reactor. Comparative analyses are carried out for $\mathrm{BN}$ 1200 type reactor with three different options such as

Case-1: Core with sodium plenum (Reference option);

Case-2: Core with upper axial fertile blanket (Traditional design);

Case-3: Core increased height $(100 \mathrm{~cm}$ from $85 \mathrm{~cm})$ and with sodium plenum (Combined option).

With each of the option, the sodium void reactivity effect is $0.5 \% \mathrm{dk} / \mathrm{k}, 1.9 \% \mathrm{dk} / \mathrm{k}$ and $1.31 \% \mathrm{dk} / \mathrm{k}$, respec- tively. The most sever BDBA is ULOF and the results are shown in Figure $\mathbf{4}$ and summarized as follows:

- Only Case-1 resulted in power reduction and stabilized;

- The other two cases results in power excursion.

As discussed before, although the Case-1 option has technical and economical disadvantage, it is concluded that the final preference is given to Case-1 design assuming self protection of the reactor even under conditions of incredible BDBA.

\subsection{Indian Approach}

In India, there is a loop-type Fast Breeder Test Reactor (FBTR) of 13.5 MWe. FBTR is in operation since 1985, and is used as the test bed for FR fuels and materials. The loop-type Prototype Fast Breeder Reactor (PFBR) of $500 \mathrm{MWe}$ will start from 2013. After that Commercial Fast Breeder Reactor (CFBR) of $500 \mathrm{MWe}$ is planned to operate from 2023 [16].

As for the safety feature, analyses of CDA were performed to confirm that the energy release is under 100 MJ. The scale down test and the full scale test are performed for the safety grade decay heat removal system of PFBR and CFBR, respectively. The partial and the wholecore catchers are provided for PFBR and CFBR.

Subsequent to the Fukushima accident, a task force was constituted for reviewing the safety of PFBR against external events under the direction of Atomic Energy Regulatory Board (AERB). The task force carried out is in-depth review by forming various working groups. The scope of the task force is to check the following items [17]:

- Capability of reactor shut down, maintaining shut down condition and core cooling;

- Capability to maintain containment integrity under credible BDBA conditions;

- Affect on storage facilities of spent fuel and fresh fuel assemblies;

- Issue related to storage of large quantity of sodium;

- Radiological impact at site and public domain;

- Accident management strategy and emergency preparedness;

- Availability of diesel generator beyond design basis scenario.

\subsection{Chinese Approach}

CEFR has achieved its first critical on July 2010, and connected to grid on July 2011. During 2011-2012 the conceptual design of CFR-1000 is on-going, and from 2012 the conceptual design of CFR-600 is under-planning. The CFR-1000 is a 1000 MWe SFR and it has a negative reactivity feedback [18].

After the Fukushima accident, the China government 

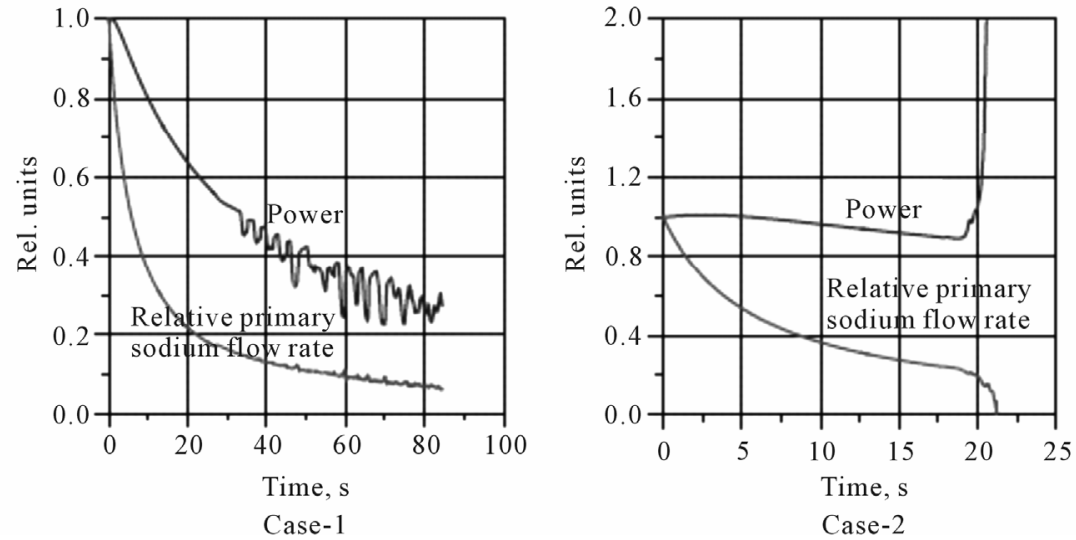

(a)
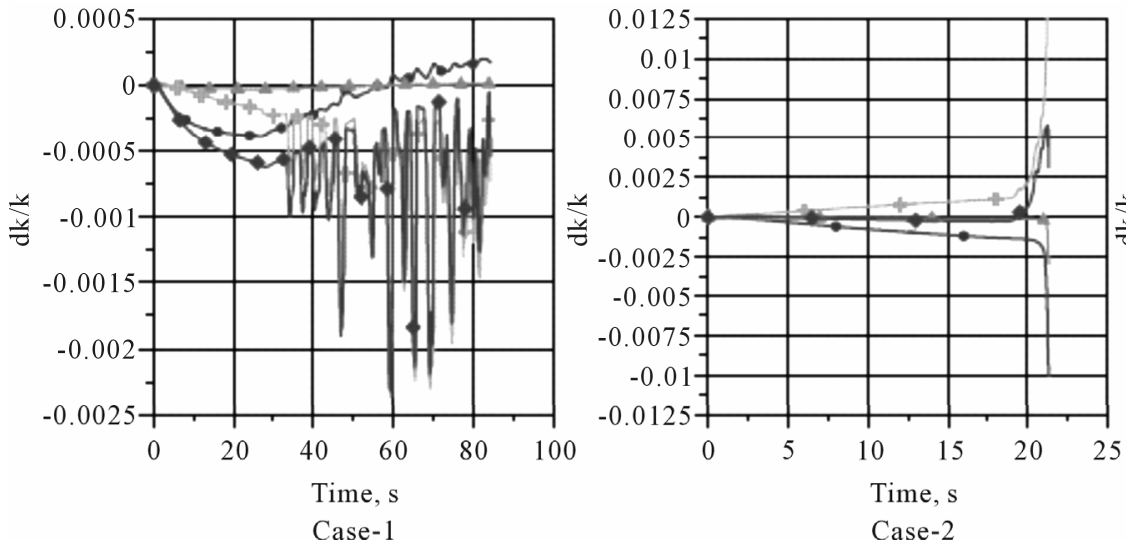

(b)
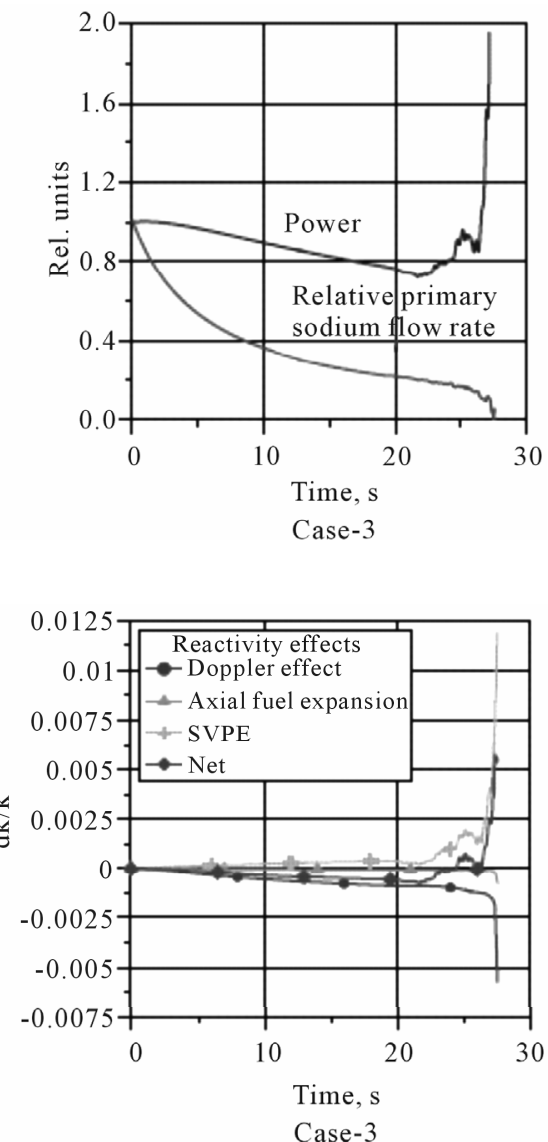

Case-3

Figure 4. Behavior of reactor parameters, ULOF accident [14,15]. (a) Relative power and primary sodium flow rates; (b) Reactivity effects.

decided to organize a comprehensive safety inspection system to nuclear facilities immediately, to strengthen management of the security of existing nuclear facilities, to conduct a thorough review of NPPs under construction with the most advanced standards, and to prepare the nuclear security plan immediately and suspend approvals of all of the new NPP projects [19].

\subsection{United States Approach}

In the aftermath of Fukushima accident, the importance of passive decay heat removal capability of advanced reactor designs has been emphasized world-wide. In the US, the potential of SFRs to survive severe accident initiators with no core damage has been demonstrated during extensive testing programs with EBR-II and the Fast Flux Test Facility (FFTF). US approach is to rely on inherent and passive safety measures to reduce the likelihood of severe accidents to a level that they belong in residual risk category and can be handled with the DiD considerations with adequate emergency planning [20].

Emphasis is on severe accident prevention, not on mitigation. Inherent safety concepts, including favorable reactivity feedback, natural circulation cooling, and design choices resulting in favorable dispersive characteristics for failed fuel, can be used to increase the level of safety to the point where it is highly unlikely, or perhaps even not credible, for such severe accident consequences to occur [21].

\section{IAEA'S ACTIVITIES FOR SFR}

The main activities of IAEA on fast reactors are the organization of regular topical technical meetings for in-depth information exchange related to development, design, construction and operation of fast rector plants, the organization of large conferences such as FR09, establishment of a forum for broad exchanges on technical requirements for Generation-IV fast reactor systems with emphasis on safety and related issues, to carry out coordinated research projects of common interest to the Technical Working Group on Fast Reactors (TWG-FR) member states, to secure training and education, and to provide support to IAEA nuclear safety and security department for preparation of fast rector standards, etc. [22]. 
Among workshop activities, GIF-IAEA/INPRO workshop concluded, on the safety aspect of SFR, that the following technical provisions in the future SFRs are important [23]:

- High reliability of the Reactor Shut-down System (RSS) based on two independent active RSS and one additional passive RSS;

- Maintain coolant level in reactor vessel even in Design Extension Conditions (DEC);

- Diversified and passive decay heat removal systems able to cool the core even in coolant leak conditions;

- Seismic protection devices;

- No impact of sodium leak on the containment vessel in Design Basis Accidents (DBA) and reduced impact in case of extreme/severe DECs;

- No energetic consequences in case of core disruptive accident through a combination of prevention and mitigation;

- Improvement of performances and Verification, Validation and Quantification (V\&V\&Q) of modeling and simulation tools for design and safety analyses of innovative SFRs.

IAEA encourages the improvement of modeling, simulation and V\&V\&Q, and thus proposed several benchmark problems to validate the accuracy of calculation tools, such as the tests of control rod withdrawal and sodium natural circulation both performed during PHENIX end of life test, the sodium natural convection test in the upper plenum of Monju, and the EBR-II shut down heat removal test.

Also several Technical Meetings (TM) have been held in the field of FR safety. Among them, the main conclusions of the TM on "Impact of Fukushima Event on FR Designs" are the importance of harmonization of the safety approaches in different countries, development of a common safety standard, installation of diversified and passive safety systems, reevaluation of seismicity, advanced modeling and simulation, and development of suitable monitoring instrumentation under severe accident conditions [24].

\section{CONCLUSIONS}

The safety aspects of FRs have been reviewed in representative countries which have developed or have a plan to develop fast reactors in near future, especially after the Fukushima accident on March 11, 2011. These countries are improving the safety of SFRs by considering the DiD. The designs of SFRs should have tolerance to DBA and BDBA caused by internal and external events. The inherent safety and passive safety should be effectively utilized for reactor shut down and reactor cooling. For the case of severe accidents, it is indispensable first to shut-down reactors. Therefore, diversified control equipments should be considered for criticality control with passive control if possible. Furthermore, decay heat removal is also indispensable even when the case of SBO. For SFRs, natural circulation can be expected in the sodium heat transport systems and the decay heat can be removal to atmosphere by the air cooling system. This is the case when sodium is maintained above the specified height. So it is important to install guard-vessel or double wall tube in the sodium heat transport systems.

Also, for Anticipated Transient without Scram (ATWS) especially ULOF, sodium boiling occurs due to the mismatch in power and flow. Such a case, sodium reactivity effect should be small, and negative if possible. Of course, many ideas to make the sodium reactivity effect negative deteriorate the breeding characteristics of reactor core. Therefore, it is important to find out most suitable idea.

The sodium reactivity effect is negative for small or medium sized reactors because of the large neutron leakage from the core in case of sodium voiding. We believe, therefore, that we have chance to keep the negative sodium reactivity effect by using small or medium sized cores. However, for the case of large core, safety design enhancement with heterogeneous structure or with sodium upper-plenum above the core would be inevitable to achieve the negative or small positive sodium reactivity effect. This type of design in small, medium or large SFR makes energy release mild even in ULOF accident.

\section{REFERENCES}

[1] Nakai, R. (2009) Design and assessment approach on advanced SFR safety with emphasis on the core disruptive accident issue. Proceedings of International Conference on Fast Reactor and Related Fuel Cycles (FR09), Kyoto, 7-11 December 2009.

[2] Konomura, M. (2012) Severe accident countermeasures of SFR (on Monju). Proceedings of JAEA-IAEA International Workshop on Prevention and Mitigation of Severe Accidents in Sodium-Cooled Fast Reactors, Tsuruga, 11-13 June 2012.

[3] Sofu, T. (2012) Importance of Passive safety and role of Monju for the future SFRs. Proceedings of JAEA-IAEA International Workshop on Prevention and Mitigation of Severe Accidents in Sodium-Cooled Fast Reactors, Tsuruga, 11-13 June 2012.

[4] Nakai, R. (2012) Safety approach based on basic safety characteristics of SFR; harmonization of safety design criteria. Proceedings of JAEA-IAEA International Workshop on Prevention and Mitigation of Severe Accidents in Sodium-Cooled Fast Reactors, Tsuruga, 11-13 June 2012.

[5] Kubo, S., Shimakawa, Y., Yamano, H. and Kotake, S. (2011) Safety design requirements for safety systems and components of JSFR. Journal of Nuclear Science and Technology, 48, 547-555. 
doi:10.1080/18811248.2011.9711732

[6] Aoto, K., Uto, N., Sakamoto, Y., Ito, T., Toda, M. and Kotake, S. (2011) Design study and R \& D progress on Japan sodium-cooled fast reactor. Journal of Nuclear Science and Technology, 48, 463-471. doi:10.1080/18811248.2011.9711720

[7] Sato, I., Tobita, Y., Konishi, K., Kamiyama, K., Toyooka, J., Nakai, R., Kubo, S., Kotake, S., Koyama, K., Vassiliev, Y., Vurim, A., Zuev, V. and Kolodeshnikov, A. (2011) Safety strategy of JSFR eliminating severe recriticality events and establishing in-vessel retention in the core disruptive accident. Journal of Nuclear Science and Technology, 48, 556-566.

doi:10.1080/18811248.2011.9711733

[8] IAEA (2005) Status of innovative small and medium sized reactor designs. IAEA TECDOC-1485.

[9] IAEA (2007) Status of small reactor designs without onsite refueling. IAEA TECDOC-1536, 2, 395-444.

[10] Beils, S., Carluec, B., Devictor, N., Fiorini, G.L. and Sauvage, J.F. (2011) Safety approach and R \& D program for future French sodium-cooled fast reactors. Journal of Nuclear Science and Technology, 48, 510-515. doi:10.1080/18811248.2011.9711727

[11] Palmiotti, G., Salvatores, M. and Assawaroongruengchot, M. (2011) Impact of the core minor actinide content on fast reactor reactivity coefficients. Journal of Nuclear Science and Technology, 48, 628-634. doi:10.1080/18811248.2011.9711743

[12] Carluec, B., Lo Point, P., Mariteau, P. and Capelle, S. (2012) Severe accident countermeasure of SFR. Proceedings of JAEA-IAEA International Workshop on Prevention and Mitigation of Severe Accidents in Sodium-Cooled Fast Reactors, Tsuruga, 11-13 June 2012.

[13] International Nuclear Safety Advisory Group (1996) Defense-in-depth in nuclear safety. INSAG Series 10, IAEA, Vienna.

[14] Shvetsov, I. (2012) Russian safety approach for NPPs with SFR. Proceedings of JAEA-IAEA International Workshop on Prevention and Mitigation of Severe Accidents in Sodium-Cooled Fast Reactors, Tsuruga, 11-13 June 2012.

[15] Poplavsky, V.M., Matveev, V.I., Eliseev, V.A., Kuznetsov, I.A., Volkov, A.V., Shvetsov, Y.E., Khomyakov, Y.S. and Tsiboulia, A.M. (2011) Studies on influence of sodium void reactivity effect on the concept of the core and safety of advanced fast reactor. Journal of Nuclear Science and Technology, 48, 538-546. doi:10.1080/18811248.2011.9711731

[16] Chellapandi, P. (2012) Overview of Indian FBR programme. Proceedings of JAEA-IAEA International Workshop on Prevention and Mitigation of Severe Accidents in Sodium-Cooled Fast Reactors, Tsuruga, 11-13 June 2012.

[17] Kumar, P. (2012) Proposed activities at PFBR in the wake of Fukushima accident. Proceedings of JAEA-IAEA International Workshop on Prevention and Mitigation of Severe Accidents in Sodium-Cooled Fast Reactors, Tsuruga, 11-13 June 2012.

[18] Ren, L. (2012) Status of China National SFR Program. Proceedings of JAEA-IAEA International Workshop on Prevention and Mitigation of Severe Accidents in Sodium-Cooled Fast Reactors, Tsuruga, 11-13 June 2012.

[19] Ren, L. (2012) Severe accidents analysis in CEFR and technology gaps. Proceedings of JAEA-IAEA International Workshop on Prevention and Mitigation of Severe Accidents in Sodium-Cooled Fast Reactors, Tsuruga, 11-13 June 2012.

[20] Sofu, T. (2012) US approach for inherent prevention and mitigation of SFR severe accidents. Proceedings of JAEAIAEA International Workshop on Prevention and Mitigation of Severe Accidents in Sodium-Cooled Fast Reactors, Tsuruga, 11-13 June 2012.

[21] Wigeland, R.A. and Cahalan, J.E. (2011) Inherent prevention and mitigation of severe accident consequences in sodium-cooled fast reactors. Journal of Nuclear Science and Technology, 48, 516-523. doi:10.1080/18811248.2011.9711728

[22] Bychkov, A. (2012) Fast reactors and closed fuel cycle: Safety technologies and sustainability. Proceedings of JAEA-IAEA International Workshop on Prevention and Mitigation of Severe Accidents in Sodium-Cooled Fast Reactors, Tsuruga, 11-13 June 2012.

[23] IAEA (2011) GIF-IAEA/INPRO Workshop on Safety Aspects of Sodium-Cooled Fast Reactors, IAEA, Vienna.

[24] IAEA/INPRO (2012) Technical meeting on impact of Fukushima event on current and future fast reactor designs: The INPRO methodology and collaborative projects related to fast reactor cooling. Hermholtz Zentrum Dresden Rossendorf (HZDR), IAEA, Vienna. 